\title{
The Relationships Between Determination of Treatment Success and Emotional Factors in Patients With Chronic Musculoskeletal Pain
}

\author{
Tae Im Yi, MD, Bo Kyoung Kim, MD, Seung A Ha, MD, Ji Young Lim, MA \\ Department of Rehabilitation Medicine, Bundang Jesaeng General Hospital, Seongnam, Korea
}

\begin{abstract}
Objective To investigate the relationship of the patient's criteria of successful treatment to emotional factors in patients with chronic musculoskeletal pain.

Methods Patients who visited our outpatient hospital due to chronic musculoskeletal pain were evaluated using a questionnaire survey. Patients were evaluated with the Patient-Centered Outcomes Questionnaire (PCOQ) to investigate their expectation and criteria for success regarding treatment of chronic musculoskeletal pain. Beck Depression Inventory and State-Trait Anxiety Inventory were used to check for psychological variables. Correlations among each of the variables were evaluated statistically.

Results Patients with higher levels of depression and anxiety needed larger improvements to consider the treatment as a success in the pain domain (depression, $r=0.398, p=0.04$; anxiety, $r=0.447, p=0.02$ ) and emotional distress domain (depression, $\mathrm{r}=0.617, \mathrm{p}=0.001$; anxiety, $\mathrm{r}=0.415, \mathrm{p}=0.03$ ), but had lower level of expectation of the treatment in the pain domain (depression, $\mathrm{r}=-0.427, \mathrm{p}=0.01$; anxiety, $\mathrm{r}=-0.441, \mathrm{p}=0.004$ ), emotional distress domain (depression, $\mathrm{r}=-0.454, \mathrm{p}=0.01$; anxiety, $\mathrm{r}=-0.395, \mathrm{p}=0.04$ ), and interference of daily activities domain (depression, $\mathrm{r}=-0.474, \mathrm{p}=0.01$; anxiety, $\mathrm{r}=-0.396, \mathrm{p}=0.04$ ). Patients were classified into 3 clusters based on the importance rating of each domain via a hierarchical analysis. The cluster of the patients with the higher rating of importance across all domains (importance of pain domain, 9.54; fatigue domain, 9.08; emotional distress domain, 9.23; interference of daily activities domain, 9.23) had the highest level of depression and anxiety.

Conclusion Consideration of psychological factors, especially in patients who require larger improvements in all treatment domains, may be helpful for the successful treatment of chronic musculoskeletal pain.
\end{abstract}

Keywords Chronic pain, Questionnaire, Depression, Anxiety

Received April 23, 2013; Accepted September 17, 2013

Corresponding author: Bo Kyoung Kim

Department of Rehabilitation Medicine, Bundang Jesaeng General Hospital, 20 Seohyeon-ro 180beon-gil, Bundang-gu, Seongnam 463-774, Korea

Tel: +82-31-779-0395, Fax: +82-31-779-0635, E-mail: equoo@naver.com

(c) This is an open-access article distributed under the terms of the Creative Commons Attribution Non-Commercial License (http://creativecommons. org/licenses/by-nc/3.0) which permits unrestricted noncommercial use, distribution, and reproduction in any medium, provided the original work is properly cited.

Copyright $\odot 2014$ by Korean Academy of Rehabilitation Medicine

\section{INTRODUCTION}

It is known that patients with chronic musculoskeletal pain may have different perspectives on successful treatment than those of clinicians [1-4]. From previous studies, we found that patients do not expect their treatment to be successful [2-5], that they need a greater improvement to consider treatment successful compared to clinicians [2-4], and that post-treatment improvement 
does not meet patient's criteria of successful treatment [6]. Since patients with chronic musculoskeletal pain come to see clinicians presenting various symptoms and attempting to achieve differing goals, such as emotional discomfort, stress, and interference of daily activities as well as their pain [7], it is important to determine successful treatment from the perspective of patients and to identify factors that can influence that success.

The Patient-Centered Outcomes Questionnaire (PCOQ) was developed to measure the criteria for success and expectations for treatment from the patients' perspectives [3]. The PCOQ asks patients to evaluate the usual level, criteria of successful treatment, and expectation to the treatment in 4 different domains, such as pain, fatigue, emotional distress, and interference of daily activities. Also, patients are asked to rate how important it is for them to see improvements in each domain to evaluate the importance of the four domains.

Though there have been many studies on various treatments for reducing chronic musculoskeletal pain and factors influencing that pain, such studies are based on statistically significant changes from the perspective of clinicians. There are few studies that investigated successful treatment from perspective of patients $[2-6,8]$. Thus, the purpose of this article is to use the PCOQ to investigate the relationship between patients' expectation to treatment, their criteria for successful treatment in each domain, and emotional factors including depression and anxiety.

\section{MATERIALS AND METHODS}

\section{Participants}

Participants were 56 patients who visited the Department of Rehabilitation Medicine at our hospital with chronic musculoskeletal pain as the chief complaint from May to July of 2012. The musculoskeletal pain is limited to pain occurring primarily due to musculoskeletal origin, and chronic pain is defined as pain that lasted for 6 months or longer. Radiologic evaluation, blood test, and electrophysiologic diagnosis necessary for diagnosing each patient were conducted.

Two patients were aged less than 20 and more than 79 , one patient whose pain was primarily caused by the central nervous system, two patients who had had neurosurgery or orthopedic surgery within 6 months, and one patient who did not fully complete the questionnaire were excluded. Therefore, a total of 50 subjects including 22 males and 28 females were used for data collection. The average age was 51.2 \pm 11.4 years and the average duration of pain was $47 \pm 73.1$ months (Table 1). The average level of pain on a visual analogue scale was $6.1 \pm 1$.6. As for the pain sites, the number cases with occurrences of foot pain and lumbar pain were 12, those of shoulder and posterior neck pain were 10 , those of knee pain were 5 , and those of hip pain were 3 . Of these, 2 cases involved 2 pain sites.

\section{Study methods}

\section{Survey overview}

This was a cross-sectional study, which used the Beck Depression Inventory (BDI), the State-Trait Anxiety In-

Table 1. Participants' demographics and descriptive statistics of PCOQ ( $n=50)$

\begin{tabular}{|cc}
\hline \multicolumn{1}{c}{ Characteristic } & Value \\
\hline Sex (male:female) & $22: 28$ \\
\hline Age (yr) & $51.2 \pm 11.4$ \\
\hline Durations (mo) & $47.0 \pm 73.1$ \\
\hline Usual & $6.1 \pm 1.5$ \\
\hline Pain & $5.6 \pm 2.5$ \\
\hline Fatigue & $6.1 \pm 2.1$ \\
\hline Emotional distress & $5.0 \pm 2.3$ \\
\hline Interference of daily activities & \\
\hline Success & $1.4 \pm 0.9$ \\
\hline Pain & $1.6 \pm 1.0$ \\
\hline Fatigue & $1.4 \pm 0.8$ \\
\hline Emotional distress & $1.0 \pm 0.8$ \\
\hline Interference of daily activities & \\
\hline Expected & $3.4 \pm 1.3$ \\
\hline Pain & $3.5 \pm 1.6$ \\
\hline Fatigue & $2.9 \pm 1.3$ \\
\hline Emotional distress & $2.7 \pm 1.1$ \\
\hline Interference of daily activities & \\
\hline Importance & $9.3 \pm 1.2$ \\
\hline Pain & $6.7 \pm 3.2$ \\
\hline Fatigue & $6.2 \pm 3.5$ \\
\hline Emotional distress & $6.6 \pm 3.5$ \\
\hline Interference of daily activities & \\
\hline Var & \\
\hline
\end{tabular}

Values are presented as mean \pm standard deviation. PCOQ, Patient-Centered Outcomes Questionnaire. 
ventory (STAI), and the PCOQ when a patient with chronic musculoskeletal pain whose duration of disease was 6 months or longer first visited the institution. The survey was conducted in a separately arranged room where the patient was guided to fill out the paper-based questionnaire.

\section{Survey methodology}

Patient-Centered Outcomes Questionnaire: The PCOQ, developed by Robinson et al. [3], was translated into Korean by the current author. This questionnaire consisted of 4 chronic pain-related domains, such as pain, fatigue, emotional distress, and interference of daily activities, and it was designed to evaluate the usual level, ideal state, criteria of successful treatment, and expectation of treatment in each domain. In this questionnaire, the criteria of successful treatment was meant to identify to what extent the condition needed to improve after the treatment in order to be considered successful, and the expectation of treatment was meant to identify to what extent the condition is expected to improve. Patients were asked to score each item on a scale from 0 (none) to 10 (the most serious discomfort one can imagine). Also, the degrees of importance of each domain were scored from 0 (no importance at all) to 10 (the most important).

Beck depression inventory: The BDI, developed by Beck et al. [9] and translated by Han et al. [10], is used for this study. This inventory consisted of a total of 21 questions with a 4 point scale from 0 to 3 , and it was used to evaluate sub-items for diagnosing depression, such as sad feelings, reduction of pleasure, guilt, hopelessness, sleep disorder, suicidal ideation, reduction in desire, etc. [11].

State-Trait Anxiety Index: The STAI was developed by Spielberger, and Kim and Shin [12] translated it into Korean. The STAI consisted of a total of 40 questions, of which 20 questions are used to measure the state anxiety felt at the moment of the survey, and the other 20 questions measure trait anxiety felt generally in daily life. Each question was scored on a 4 point scale from 1 (not at all) to 4 (strongly so) [11]. The state anxiety is defined as a human emotion or state that varies in strength over time, and the level of state anxiety increases when one perceives a certain environment as threatening regardless of objective danger [12]. The trait anxiety index measures personal differences in tendency for anxiety [13]. This study used only the state anxiety index (STAI-S) to assess the patients' current state.

Statistical analysis: The SPSS 18.0 program for Windows (SPSS Inc., Chicago, IL, USA) was used for statistics. Nonparametric statistics were used since the numbers of the sample size were small. Spearman rank correlation coefficients were used to evaluate the interrelations among the PCOQ, BDI, and STAI-S, and hierarchical agglomerative cluster analysis was used to classify the subjects into clusters by the importance of each domain. Also, the Kruskal-Wallis test was used to examine if there were differences in the importance and in the levels of depression and anxiety among the clusters, and then the Mann-Whitney test was performed for post-hoc test. The significance level was defined as $\mathrm{p} \leq 0.05$.

\section{RESULTS}

\section{The PCOQ, BDI, and STAI-S}

Participants' demographics and descriptive statistics from the PCOQ are presented in Table 1. From the PCOQ, the average score of pain was 6.1, that of fatigue was 5.6, that of emotional stress was 6.12 , and that of interference of daily activities was 5.0. The average score of an ideal state in each domain was 0.1 . As the average scores of the criteria of successful treatment were 1.4, 1.6, 1.4, and 1.0 in each respective domain, and those of the expectation of treatment were $3.4,3.5,2.9$, and 2.7, respectively. It turned out that the expectation for the treatment did not meet the criteria of successful treatment. The average scores of importance in individual domain were 9.3, 6.7, 6.2 , and 6.6, respectively (Table 1). The average BDI score was 13.0 and the average STAI-S score was 41.9.

Analysis of the interrelations among the PCOQ, BDI, and STAI-S

The BDI showed significant correlations with usual level of pain ( $r=0.557, p=0.003)$, fatigue $(r=0.697, p=0.001)$, emotional distress $(\mathrm{r}=0.845, \mathrm{p}=0.001)$, and interference of daily activities $(\mathrm{r}=0.614, \mathrm{p}=0.001)$. The STAI showed significant correlations with usual level of pain ( $\mathrm{r}=0.526$, $\mathrm{p}=0.006)$, emotional distress ( $\mathrm{r}=0.415, \mathrm{p}=0.03)$, and interference of daily activities $(r=0.394, p=0.04)$ (Table 2$)$.

The BDI and STAI-S show a negative correlation with the criteria for successful treatment in all domains of PCOQ, but that correlation is not statistically significant. Since patients with higher BDI and STAI-S have higher 
scores of the usual level in the PCOQ domain, even though same amount of discomfort is lower relative to that of patients with lower BDI and STAI-S, patients with higher BDI and STAI-S might not consider the treatment to be successful. To evaluate the amount of improvement to consider the treatment as successful, we used values obtained by subtracting the criteria of successful treatment from the usual level. The average value obtained by subtracting the criteria of successful treatment from the usual level were $4.76 \pm 1.53$ for pain, $4.00 \pm 2.21$ for fatigue, $4.76 \pm 2.14$ for emotional distress, and $4.20 \pm 2.02$ for interference of daily activities. The BDI showed significant correlations with values obtained by subtracting the criteria of successful treatment from usual level in pain ( $\mathrm{r}=0.398, \mathrm{p}=0.04)$, fatigue $(\mathrm{r}=0.657, \mathrm{p}=0.001)$, emotional distress $(\mathrm{r}=0.617, \mathrm{p}=0.001)$, and interference of daily activities ( $\mathrm{r}=0.558, \mathrm{p}=0.002$ ). The STAI-S showed significant correlations with values obtained by subtracting the criteria of successful treatment from usual level in pain $(\mathrm{r}=0.447, \mathrm{p}=0.02)$ and emotional distress $(\mathrm{r}=0.415$, $\mathrm{p}=0.03$ ) (Table 2). We found that patients with higher BDI

Table 2. The correlation between depression and anxiety and the four domains of PCOQ; usual, success, and expected level of pain, fatigue, emotional distress, and interference of daily activities

\begin{tabular}{lll}
\hline & BDI & STAI-S \\
\hline Usual & & \\
\hline Pain & $0.557^{*}$ & $0.526^{*}$ \\
\hline Fatigue & $0.697^{*}$ & 0.060 \\
\hline Emotional distress & $0.845^{*}$ & $0.415^{*}$ \\
\hline Interference with daily activities & $0.614^{*}$ & $0.394^{*}$ \\
\hline Success & & \\
\hline Pain & $0.398^{*}$ & $0.447^{*}$ \\
\hline Fatigue & $0.657^{*}$ & 0.109 \\
\hline Emotional distress & $0.617^{*}$ & $0.415^{*}$ \\
\hline Interference with daily activities & $0.588^{*}$ & 0.374 \\
\hline Expected & & \\
\hline Pain & $-0.427^{*}$ & $-0.441^{*}$ \\
\hline Fatigue & $-0.394^{*}$ & -0.209 \\
\hline Emotional distress & $-0.454^{*}$ & $-0.395^{*}$ \\
\hline Interference with daily activities & $-0.474^{*}$ & $-0.396^{*}$ \\
\hline
\end{tabular}

Values are Spearman rank correlation coefficients. PCOQ, patient-centered outcomes questionnaire; BDI, Beck Depression Inventory; STAI-S, state anxiety of StateTrait Anxiety Inventory. ${ }^{*} \mathrm{p}<0.05$. and STAI-S scores need larger reduction in pain, fatigue, emotional distress, and interference of daily activities to consider the treatment to be successful.

The BDI and STAI-S significantly correlate with the expectation of the treatment in all domains of PCOQ. However, as mentioned above, patients with higher BDI and STAI-S have higher scores of the usual levels in PCOQ domain, so even though those patients expect the same amount of reduction in discomfort, the results can show the patients with higher BDI and STAI-S to have lower levels of expectation to the treatment. To evaluate the amount of improvement patients expect from the treatment, we used values obtained by subtracting the expected level from usual level. The average value obtained by subtracting the expected level from usual level was $3.48 \pm 1.58$ for pain, $3.16 \pm 1.88$ for fatigue, $3.52 \pm 1.19$ for emotional distress, and $2.52 \pm 1.75$ for interference of daily activities. The BDI showed significant negative correlations with values obtained by subtracting the expected level from the usual level in pain $(r=-0.427, p=0.01)$, fatigue $(\mathrm{r}=-0.394, \mathrm{p}=0.005)$, emotional distress $(\mathrm{r}=-0.454$, $\mathrm{p}=0.01)$, and interference of daily activities $(\mathrm{r}=-0.474$, $\mathrm{p}=0.01$ ). The STAI-S showed significant negative correlations with values obtained by subtracting the expected level from the usual level in pain ( $\mathrm{r}=-0.441, \mathrm{p}=0.004)$, emotional distress $(r=-0.395, p=0.04)$, and interference

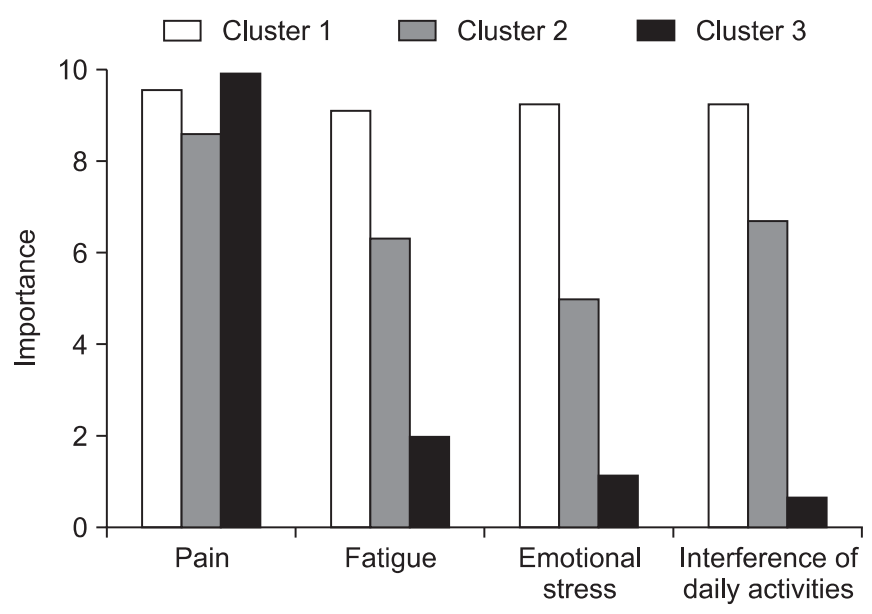

Fig. 1. Patients were classified into the 3 clusters based on the importance rating of each domain by hierarchical analysis. Cluster 1, patients with the high importance across all domains; Cluster 2, patients with moderately high importance in domains except pain domain; Cluster 3 , patients with low importance in domains except pain domain. 
of daily activities $(\mathrm{r}=-0.396, \mathrm{p}=0.04)$ (Table 2$)$. We found that higher BDI and STAI-S scores correlate with lower levels of expectations for the treatment.

Patients were classified into 3 clusters based on the importance rating of each domain found through hierarchical cluster analysis. The first cluster consisted of 24 patients whose score for pain was 9.54, while their score for fatigue was 9.08, that of emotional distress was 9.23, and that of interference of daily activities was 9.23 . The second cluster included 14 patients with a score for pain of 8.57, that for fatigue of 6.29 , that for emotional distress of 5.00, and that for interference of daily activities of 6.71. The third cluster consisted of 12 patients with a score for pain of 9.87, that for fatigue of 2.00 , that for emotional distress of 1.17, and that for interference of daily activities of 0.67 (Fig. 1). The Kruskal-Wallis non-parametric test was conducted to clarify whether there were differences in importance of pain, fatigue, emotional distress, and interference of daily activities among the clusters. There were no significant differences in pain among the clusters, with $\mathrm{p}=0.148$, but in the other domains, there were significant difference among the clusters, with $\mathrm{p}=0.001$. As a result of the post-hoc Mann-Whitney test, there were mutually significant differences among the three clusters in the importance of fatigue, emotional distress, and interference of daily activities (Table 3 ). Since the importance of pain was high in all the clusters, the first cluster can be characterized to have high importance in all domains, the second as that of moderate importance in domains except for the pain domain, and the third as that of low importance in domains except pain domain. The possible differences in the BDI and STAI-S among the clusters was examined through Kruskal-Wallis and Mann-Whitney post-hoc tests. The Kruskal-Wallis test showed that there were significant differences in the BDI and STAI-S among the clusters (depression, $\mathrm{p}=0.009$; anxiety, $\mathrm{p}=0.16$ ), and the Mann-Whitney post-hoc test revealed that there were significant differences in both the BDI and STAI-S between the first and second clusters (depression, $\mathrm{p}=0.01$; anxiety, $\mathrm{p}=0.007$ ) and also between the first and the third clusters (depression, $\mathrm{p}=0.01$; anxiety, $\mathrm{p}=0.04)$. However, there were no significant differ-

Table 3. The difference between 3 clusters in importance of four domains of PCOQ

\begin{tabular}{lcccc}
\hline & Pain & Fatigue & Emotional distress & Interference of daily activities \\
\hline Cluster 1-2 & 0.467 & 0.001 & 0.001 & 0.001 \\
Cluster 2-3 & 0.366 & 0.014 & 0.022 & 0.001 \\
Cluster 1-3 & 0.643 & 0.002 & 0.001 & 0.001 \\
\hline
\end{tabular}

Values are p-values of Mann-Whitney U test.

PCOQ, patient-centered outcomes questionnaire.

(A)

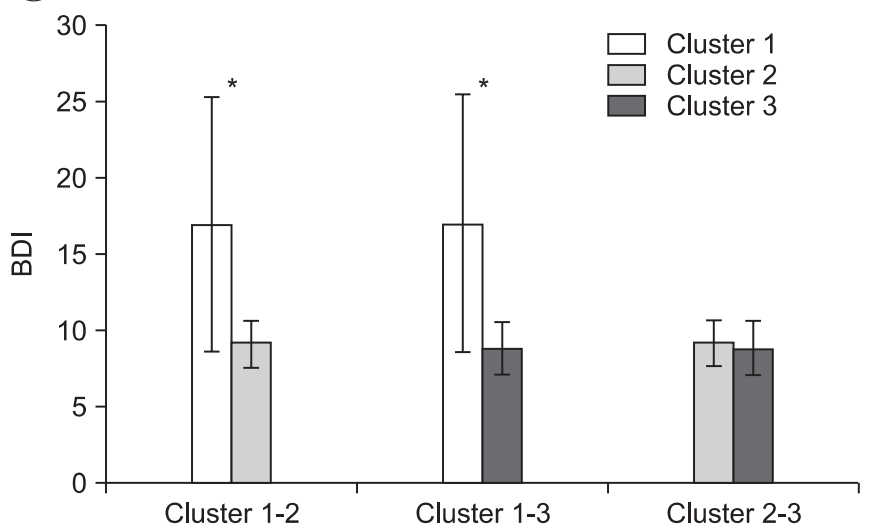

(B)

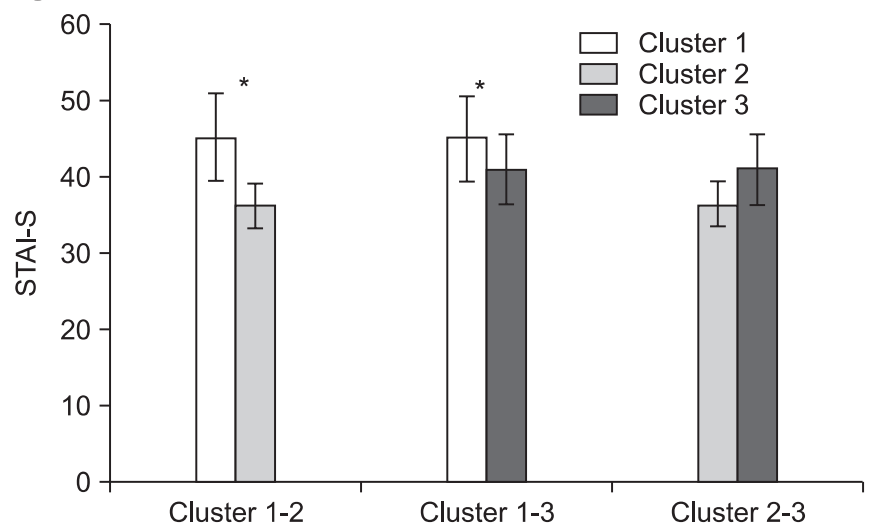

Fig. 2. Comparison of the Beck Depression Inventory (BDI, A) and state anxiety of State-Trait Anxiety Inventory (STAI$\mathrm{S}, \mathrm{B}$ ) between the patient clusters classified by rating the importance across each domain. Cluster 1, patients with the higher importance across all domains, had significantly higher levels of depression and anxiety relative to other clusters. Bars represent standard deviation. ${ }^{*} \mathrm{p}<0.05$. 
ences in the BDI and STAI-S between the second and third clusters. The first cluster, on the other hand, with high importance in all domains, has significantly higher BDI and STAI-S than the other two clusters (Fig. 2).

\section{DISCUSSION}

This study was an investigation into the relationship between the criteria for success, expectation of treatment, and levels of depression and anxiety in patients with chronic musculoskeletal pain. It was performed by quantifying the criteria and expectation of the treatment in each of the domains of pain, fatigue, emotional distress, and interference of daily activities in terms of the PCOQ. This study shows that patients' usual levels of pain, emotional distress, and interference of daily activities correlate with their levels of depression and anxiety, and that fatigue was related only to the level of depression. This is consistent with results of previous studies that showed depression and anxiety had an influence not only in pain [14] and stress [15], but also on quality of life [11]. The relationship between fatigue and the levels of depression is interpreted as the fatigue being a symptom of the depression. Afari and Buchwald [16] reviewed chronic fatigue syndrome in 2003 and suggested that chronic fatigue and anxiety overlapped in the general population, but this simple comorbidity does not mean that chronic fatigue syndrome is a physical manifestation of anxiety disorder. Studies focusing on the relationship between anxiety and fatigue in patients with chronic musculoskeletal pain have mainly paid attention to patients with fibromyalgia. Kurtze et al. [17] reported in 1998 that when dividing subjects into the groups with high depression and anxiety and with low depression and anxiety, since fibromyalgia is accompanied by depression and anxiety, the group with high depression and anxiety had high fatigue. Subsequently, a study on the influence of depression and anxiety on individual patterns of pain in fibromyalgia patients in 2001 suggested that anxiety had no significant correlation with fatigue [18]. Fishbain et al. [19] investigated factors influencing fatigue in patients with chronic low back pain and chronic neck pain in 2004, and research showed that anxiety did not have an influence on fatigue. In this study of patients with chronic musculoskeletal pain as its subjects, we did not find a relationship between fatigue and anxiety, and it is believed that further research is necessary in the relationship between anxiety and fatigue in patients with chronic musculoskeletal pain.

McCracken et al. [20] reported that persistence of symptoms was related to treatment satisfaction, and the depression symptoms showed a negative correlation with treatment satisfaction, which, though for unclear causality, suggested a relation between depressive symptoms and treatment satisfaction. However, they failed to prove a correlation between pain reduction and treatment satisfaction. In this study, patients with chronic musculoskeletal pain tended to require a larger improvement of pain to judge the treatment to be a success but had lower expectations for the treatment as they had higher levels of depression and anxiety. Thus, it is suggested that patients with chronic musculoskeletal pain patients and higher levels of depression and anxiety among other patients have difficulty in judging treatment to be successful.

This study was able to classify patients with chronic musculoskeletal pain into three clusters based on the importance of each domain in a manner consistent with Robinson et al. [3]. Zeppieri et al. [8] showed that a patient cluster with high importance on all domains had higher levels of pain, emotional distress, and interference of daily activities, and that such patients had various desires other than that of the improvement of pain. He suggested that the understanding of those desires, from the patient's perspective, would be helpful in establishing the goals of rehabilitation. In this study, we were able to find that the patient cluster with high importance on all domains was evaluated to have high levels of depression and anxiety. Thus, in such a patient cluster, it is recommended that a psychological evaluation and therapeutic approach to depression and anxiety be included.

The limitations of this study include limited information of the causality and priorities of patients' pain and other emotional elements due to the cross-sectional nature of the research. It is believed that we can get more information if further follow-up is carried out with observation of the influence of depression and anxiety on whether patients reach the criteria of successful treatment after the treatment.

In conclusion, patients with chronic musculoskeletal pain who have higher levels of depression and anxiety, among a group of patients with chronic musculoskeletal pain, have higher criteria for successful treatment. Also, 
the patient cluster with high importance in all domains of pain, fatigue, emotional distress, and interference of daily activities has higher levels of depression and anxiety. As such, the patient cluster has various needs in addition to the improvement of pain including resolution of emotional distress, restoration from fatigue, and return to daily activity, a psychological approach should be included in the planning of rehabilitative treatment.

\section{CONFLICT OF INTEREST}

No potential conflict of interest relevant to this article was reported.

\section{REFERENCES}

1. Daniel M, Long C, Murphy W, Kores R, Hutcherson W. Therapists' and chronic pain patients' perceptions of treatment outcome. J Nerv Ment Dis 1983;171:729-33.

2. Brown JL, Edwards PS, Atchison JW, Lafayette-Lucey A, Wittmer VT, Robinson ME. Defining patient-centered, multidimensional success criteria for treatment of chronic spine pain. Pain Med 2008;9:851-62.

3. Robinson ME, Brown JL, George SZ, Edwards PS, Atchison JW, Hirsh AT, et al. Multidimensional success criteria and expectations for treatment of chronic pain: the patient perspective. Pain Med 2005;6:336-45.

4. Thorne FM, Morley S. Prospective judgments of acceptable outcomes for pain, interference and activity: Patient-determined outcome criteria. Pain 2009; 144:262-9.

5. O'Brien EM, Staud RM, Hassinger AD, McCulloch RC, Craggs JG, Atchison JW, et al. Patient-centered perspective on treatment outcomes in chronic pain. Pain Med 2010;11:6-15.

6. Sanderson KB, Roditi D, George SZ, Atchison JW, Banou E, Robinson ME. Investigating patient expectations and treatment outcome in a chronic low back pain population. J Pain Res 2012;5:15-22.

7. Casarett D, Karlawish J, Sankar P, Hirschman K, Asch DA. Designing pain research from the patient's perspective: what trial end points are important to patients with chronic pain? Pain Med 2001;2:309-16.

8. Zeppieri G Jr, Lentz TA, Atchison JW, Indelicato PA, Moser MW, Vincent KR, et al. Preliminary results of patient-defined success criteria for individuals with musculoskeletal pain in outpatient physical therapy settings. Arch Phys Med Rehabil 2012;93:434-40.

9. Beck AT, Ward CH, Mendelson M, Mock J, Erbaugh J. An inventory for measuring depression. Arch Gen Psychiatry 1961;4:561-71.

10. Han HM, Yum TH, Shin YW, Kim KH, Yoon DJ, Jung GJ. A standardization study of Beck Depression Inventory in Korea. J Korean Neuropsychiatr Assoc 1986;25:487-500.

11. Lim KB, Park HJ, Kim SH, Kim DY, Kim SS. Quality of life and anxiety in patients with acute and chronic pain. J Korean Acad Rehabil Med 2010;34:752-7.

12. Kim JT, Shin DK. A study based on the standardization of the STAI for Korea. New Med J 1978;21:69-75.

13. Kim JH, Seok JH, Jon DI, Hong HJ, Hong N, Kim SJ, et al. Psychological characteristics of patients with depressive symptoms at the initial diagnosis of breast cancer: preliminary results. J Breast Cancer 2008;11:83-8.

14. Lim KB, Kim JY, Lee HJ, Kim DY, Kim JM. The relations among pain, emotional and cognitive-behavioral factors in chronic musculoskeletal pain patients. J Korean Acad Rehabil Med 2008;32:424-9.

15. Kim SJ, Kim WS, Kang YK, Lee SH, Cho SH. Influencing psychologic factors and stress in patients with chronic pain. J Korean Acad Rehabil Med 2000; 24:1161-7.

16. Afari N, Buchwald D. Chronic fatigue syndrome: a review. Am J Psychiatry 2003;160:221-36.

17. Kurtze N, Gundersen KT, Svebak S. The role of anxiety and depression in fatigue and patterns of pain among subgroups of fibromyalgia patients. Br J Med Psychol 1998;71(Pt 2):185-94.

18. Kurtze N, Svebak S. Fatigue and patterns of pain in fibromyalgia: correlations with anxiety, depression and co-morbidity in a female county sample. Br J Med Psychol 2001;74(Pt 4):523-37.

19. Fishbain DA, Cutler RB, Cole B, Lewis J, Smets E, Rosomoff HL, et al. Are patients with chronic low back pain or chronic neck pain fatigued? Pain Med 2004;5:187-95.

20. McCracken LM, Klock PA, Mingay DJ, Asbury JK, Sinclair DM. Assessment of satisfaction with treatment for chronic pain. J Pain Symptom Manage 1997;14:292-9. 